\title{
Assessment of knowledge of healthcare workers in a service hospital regarding breastfeeding practices in mothers confirmed or suspected to have COVID-19
}

\author{
A R Rajan ${ }^{1}$, Anish Kumar Vishal' ${ }^{2}$, Anupama Arvind Bhave ${ }^{3}$ \\ From ${ }^{1}$ HOD, ${ }^{3}$ Doctor, Department of Pediatrics, ${ }^{2}$ Assistant Professor, Department of Obstetrics and Gynaecology, Military Hospital Kirkee, Pune, \\ Maharashtra
}

\begin{abstract}
Introduction: Breastfeeding is nature's way of nurturing newborn and infants. Doubts exist among healthcare workers (HCWs) about the advice regarding breastfeeding to mothers who test positive/suspected for COVID-19. Objective: The objective of this study was to assess the knowledge among HCWs at a service hospital regarding breastfeeding among mothers suspected/confirmed for COVID-19. Materials and Methods: This was a hospital-based cross-sectional study. A total of 69 HCWs comprising female ward assistants, nursing assistants, nursing officers, and medical officers were included in the study. A predesigned questionnaire was used to obtain information after taking consent. Results: A total of $76.92 \%$ of female ward assistants, $57.14 \%$ of nursing assistants, $83.33 \%$ of nursing officers, and 75\% of medical officers were aware that COVID-19 is not passed through breast milk. About $92.31 \%$ of female ward assistants, $71.43 \%$ nursing assistants, $80 \%$ nursing officers, and 58.33\% medical officers knew that it is safe to give expressed breast milk from COVID-19 suspected/positive mothers. The hygiene recommendations for COVID-19 suspected/confirmed mother for breastfeeding were known to $92.31 \%$ of female ward assistants, $100 \%$ nursing assistants, $96.67 \%$ of nursing officers, and $100 \%$ of medical officers. Only $15.38 \%$ of female ward assistants, $35.71 \%$ nursing assistants, $50 \%$ of nursing officers, and $58.33 \%$ of medical officers knew not to give infant milk formula and recommended exclusive breastfeeding to a baby of COVID-19 suspected/confirmed mother. Conclusion: This study provides a foundation to focus on the promotion of optimal breastfeeding practices and creating awareness among HCWs about WHO guidance on breastfeeding during the COVID-19 pandemic.
\end{abstract}

Key words: Breastfeeding, COVID-19 suspected/positive mothers, Healthcare workers, Knowledge

$\mathrm{S}$ ARS-CoV-2 is a single-stranded RNA virus of the betacoronavirus genus. Transmission of this virus in humans is predominantly by respiratory droplets and direct contact with no conclusive evidence of vertical transmission. In recent studies, there has been no evidence of transmission of the SARSCoV-2 through breast milk. COVID-19 pandemic has been one of the biggest disruptions in the routine provision of perinatal health care worldwide. In the background of the SARS-COV-2 pandemic, there are many doubts and misconceptions amongst healthcare workers (HCWs) regarding advice to be given to mothers who are suspected/confirmed for COVID-19 regarding breastfeeding. Breastfeeding recommendations by the WHO during the COVID-19 pandemic [1] need to be disseminated to the public so as to allay the anxiety of mothers and ensure the good health of the newborn. HCWs are the best source of information for scientific knowledge in the current pandemic.

\section{Access this article online}

Received - 02 October 2020

Initial Review - 03 October 2020

Accepted - 26 November 2020

DOI: $10.32677 / \mathrm{IJCH} .2020 . v 07.111 .002$
The same doubts also exist in the minds of the mothers, which need to be addressed so that proper advice can be given to the mothers by the healthcare workers.

Exclusive breastfeeding for the first 6 months of life, followed by the continuation of breastfeeding beyond 2 years of age, is recommended by the WHO [2]. Academy of Breastfeeding Medicine [3] and Royal College of Obstetricians and Gynaecologists [4] recommend exclusive breastfeeding while observing standard respiratory and hand hygiene protocols in suspected/confirmed COVID-19 mothers. Expressed breast milk is reserved for cases where mothers are too ill to breastfeed.

The aim of this study was to assess the knowledge among HCWs at a service hospital regarding breastfeeding among mothers suspected/confirmed for COVID-19 during confinement or after delivery in the context of the COVID-19 pandemic in a hospital-based cross-sectional study. The lacunae found could be addressed, subsequently ensuring proper breastfeeding advice to mothers by HCWs.

Correspondence to: A R Rajan, Department of Pediatrics, Military Hospital Kirkee, Range Hills Road, Pune - 411 020, Maharashtra, India. E-mail: aramanrajan@gmail.com

(C) 2020 Creative Commons Attribution-NonCommercial 4.0 International License (CC BY-NC-ND 4.0). 


\section{MATERIALS AND METHODS}

This hospital-based cross-sectional study was done in a service hospital to assess the knowledge among HCWs regarding breastfeeding among mothers suspected/confirmed for COVID-19. This study was conducted in the department of pediatrics and department of obstetrics and gynecology of a service hospital in India over a period of 1 month (August 1, 2020, to August 30, 2020). Prior approval from the Institutional Ethics Committee was taken.

The case definition for suspected COVID-19 and confirmed COVID-19 was as per the WHO COVID-19 case definitions [5]. Healthcare workers, employed at the service hospital and willing to take the survey, were included in the study. Non-healthcare workers employed at the service hospital and healthcare workers unwilling for the survey were excluded from the study.

The participants were administered a peer-reviewed predesigned questionnaire regarding breastfeeding in suspected/confirmed COVID-19-positive mothers based on the WHO guidance and frequently asked questions on breastfeeding and COVID-19 for the healthcare workers [6]. Essential demographic information was collected, namely, level of seniority, name, age, sex, trade/specialty of HCW, contact address, mobile number, and educational status.

The questionnaire had 12 questions. The answering options were yes/no/do not know. The correct answer was given a score $=1$ and incorrect answer was given a score $=0$. Exceptions were two questions, which were one on breast hygiene, hand hygiene, and mask-wearing along with the cleanliness of surrounding surfaces, and second one on why breastfeeding is protective vis-a-vis social distancing advocated in all other COVID-19 suspected/confirmed cases. Each correct answer was given a score of 0.5 , and the scores were added for the final score for that question. If all correct answers were ticked, then the maximum score given was 4 and 2, respectively.

The scores of all answered questions were added to get the total score, and the maximum score was 16 . Percentage scores were derived from this data. The time taken to complete the questionnaire was $10 \mathrm{~min}$. Before this, the consent of participants was sought after assuring them of confidentiality.

This was a knowledge assessment study. The total population size of HCWs eligible for this study was approximately 80, at $99 \%$ CI with 5\% margin of error; the calculated sample size using Morgan's Table [7] was 66, but we received participation from 69 participants. The data were collected and tabulated in Microsoft Excel Sheet. Statistical analysis was done using Epi Info 7.2. Descriptive statistics, such as frequency and percentage, were calculated. The knowledge of HCWs was graded on the basis of this percentage into the following grades: $<45 \%=$ below average, $45-65 \%=$ average, $65-85 \%=$ good, and $85-100 \%=$ excellent. Question-wise awareness percentage was calculated for every category of HCW. Question-wise awareness percentage in total HCWs studied was also calculated.

\section{RESULTS}

This study included 69 HCWs as follows: 13 (18.84\%) female ward assistants, 14 (20.29\%) nursing assistants, 30 (43.48\%) nursing officers, and $12(17.39 \%)$ medical officers. About $76.92 \%$ of female ward assistants, $57.14 \%$ nursing assistants, $83.33 \%$ of nursing officers, and $75 \%$ medical officers were aware that COVID-19 is not passed through breast milk. About $92.31 \%$ of female ward assistants, $71.43 \%$ nursing assistants, $80 \%$ nursing officers, and $58.33 \%$ medical officers knew that it is safe to give evidence-based medicine (EBM) from COVID-19 suspected/ positive mothers.

The hygiene recommendations for COVID-19 suspected/ confirmed mother for breastfeeding were known to $92.31 \%$ of female ward assistants, $100 \%$ nursing assistants, $96.67 \%$ of nursing officers, and $100 \%$ of medical officers. Only $15.38 \%$ of female ward assistants, $35.71 \%$ nursing assistants, $50 \%$ of nursing officers, and $58.33 \%$ of medical officers were aware of the inadvisability of giving infant milk formula and recommended exclusive breastfeeds to a baby of COVID-19 suspected/ confirmed mother.

The knowledge about non-acceptance of free supplies of formula milk was adequate in $30.77 \%$ of female ward assistants, $50 \%$ of nursing assistants, $43.33 \%$ of nursing officers, and $66.67 \%$ of medical officers. The awareness that the breastfeeding guidelines in COVID-19 suspected/confirmed mothers were different from social distancing norms for the general population was seen in $23.08 \%$ of female ward assistants, $50 \%$ nursing assistants, $46.67 \%$ of nursing officers, and $75 \%$ of medical officers.

Healthcare workers are faced with the dilemma of separating the baby from mother and allowing the skin to skin contact of the baby with COVID-19 suspected/confirmed mother. Skin to skin contact is recommended and favored by $69.23 \%$ of female ward assistants, $42.86 \%$ nursing assistants, $76.67 \%$ nursing officers, and $66.67 \%$ medical officers.

Awareness to follow the protocol of giving alternatives in case of inability to breastfeed by COVID-19 suspected/ confirmed mother, EBM if not available (N.A.) then Donor Human Milk, if N.A. then wet-nursing (if acceptable), if N.A. then infant milk formula only if it is feasible, correctly prepared, safe, and sustainable. This was observed in $46.15 \%$ of female ward assistants, $35.71 \%$ of nursing assistants, $73.33 \%$ of nursing officers, and $66.67 \%$ of medical officers. About $84.06 \%$ of healthcare workers were aware that it is possible to restart breastfeeding on recovery from other illness in a COVID-19 suspected/confirmed mother. About $56.62 \%$ of the healthcare workers knew the duration of infectivity for implementation of the WHO recommendations on breastfeeding and COVID-19. The results are illustrated in Table 1.

On the whole, the knowledge and awareness level of HCWs regarding optimal breastfeeding practices in suspected/confirmed COVID-19 mothers as per the guidance provided by the WHO are depicted in Table 2 .

\section{DISCUSSION}

Breast milk is a complete, wholesome, safe, affordable, easily available, immunity providing, and culturally acceptable nutrition 
Table 1: Knowledge and awareness regarding breastfeeding and COVID-19 among healthcare workers

\begin{tabular}{|c|c|c|c|c|c|}
\hline \multicolumn{6}{|c|}{ Correct answers } \\
\hline Questions & FWA $(n=13)$ & NA $(n=14)$ & NO $(n=30)$ & MO $(n=12)$ & Total $(n=69)$ \\
\hline & Aware (n, \%) & Aware (n, \%) & Aware (n, \%) & Aware (n, \%) & Aware (n, \%) \\
\hline Q1 COVID-19 is not passed through breastfeeding? & $10(76.92)$ & $8(57.14)$ & $25(83.33)$ & $9(75 \%)$ & $52(75.36)$ \\
\hline $\begin{array}{l}\text { Q2 Baby should be breastfed and placed skin to skin } \\
\text { with mother }\end{array}$ & $9(69.23)$ & $6(42.86)$ & $23(76.67)$ & $8(66.67)$ & $46(66.67)$ \\
\hline Q3 Wash hands, wear face mask, disinfect surfaces & $12(92.31)$ & $14(100)$ & $29(96.67)$ & $12(100)$ & $67(97.10)$ \\
\hline Q4 To give only breast milk, no formula milk & $2(15.38)$ & $5(35.71)$ & $15(50)$ & $7(58.33)$ & $29(42.03)$ \\
\hline $\begin{array}{l}\text { Q5 Safe to give expressed breast milk from COVID-19 } \\
\text { mother? }\end{array}$ & $12(92.31)$ & $10(71.43)$ & $24(80)$ & $7(58.33)$ & $53(76.81)$ \\
\hline $\begin{array}{l}\text { Q6 Ability to restart breastfeed after recovery from other } \\
\text { illness }\end{array}$ & $12(92.31)$ & $7(50)$ & $29(96.67)$ & $10(83.33)$ & $58(84.06)$ \\
\hline $\begin{array}{l}\text { Q7 Time duration of relevance of the WHO } \\
\text { recommendations }\end{array}$ & $8(61.54)$ & $8(57.14)$ & $14(46.67)$ & $9(75 \%)$ & $39(56.62)$ \\
\hline $\begin{array}{l}\text { Q8 Difference in breastfeeding and social distancing } \\
\text { WHO recommendation }\end{array}$ & $3(23.08)$ & $7(50)$ & $14(46.67)$ & $9(75)$ & $33(47.83)$ \\
\hline $\begin{array}{l}\text { Q9 Health facility should not accept free supply Formula } \\
\text { milk }\end{array}$ & $4(30.77)$ & $7(50)$ & $13(43.33)$ & $8(66.67)$ & $32(46.38)$ \\
\hline Q10 Baby not to be separated from mother & $9(69.23)$ & $6(42.86)$ & $24(80 \%)$ & $7(58.33)$ & $46(66.67)$ \\
\hline Q11 Not to wash breast before every breastfeed & $13(100)$ & $4(28.57)$ & $3(10)$ & $4(33.33)$ & $24(34.78)$ \\
\hline $\begin{array}{l}\text { Q12 Alternatives in stepwise manner if unable to } \\
\text { breastfeed }\end{array}$ & $6(46.15)$ & $5(35.71)$ & $22(73.33)$ & $8(66.67)$ & $41(59.42)$ \\
\hline
\end{tabular}

Table 2: Knowledge and awareness level of HCWs regarding optimal breastfeeding practices in suspected/confirmed COVID-19 mothers

\begin{tabular}{|c|c|c|c|c|}
\hline \multirow[t]{2}{*}{ Trade of HCW $(n=69)$} & \multicolumn{4}{|c|}{ Knowledge and awareness level score } \\
\hline & Below average $(\%)$ & Average (\%) & Good (\%) & Excellent (\%) \\
\hline Female ward assistant $(n=13)$ & 0 & $4(30.77)$ & $9(69.23)$ & 0 \\
\hline Nursing assistant $(\mathrm{n}=14)$ & $2(14.29)$ & $7(50)$ & $5(35.71)$ & 0 \\
\hline Nursing officer $(n=30)$ & 0 & $8(26.67)$ & $17(56.67)$ & $5(16.66)$ \\
\hline Medical officer $(n=12)$ & 0 & $4(33.33)$ & $4(33.33)$ & $4(33.34)$ \\
\hline
\end{tabular}

HCWs: Healthcare workers

for newborns and infants. Exclusive breastfeeding improves the survival, growth, and development of the newborn and infant. Skin to skin contact with the mother provides necessary warmth and emotional bonding to the baby. Skin-to-skin contact and kangaroo mother care facilitate breastfeeding, which improves glycemic control, temperature control, and mother to baby bonding. In low birth weight babies, it reduces the severity of infection and mortality [1].

In the COVID-19 pandemic scenario, social distancing is the "new normal" for preventing the spread of SARS-COV-2 virus. This leads to a lot of confusion as to whether to let mother and baby dyad bond in close contact and breastfeed or not if the mother is COVID-19 suspected/confirmed. The recommendations of social distancing for the general population to reduce the prevalence of COVID-19 are different from guidance on breastfeeding in COVID-19 suspected/confirmed mothers as this reduces morbidity and mortality, improves lifelong survival, health, and development of the newborn/infant [6].

Since the benefit of breastfeeding outweighs the risk of COVID-19 transmission, newborns being at lower risk of COVID19 infection and active COVID-19 not detected to be transmitted through breast milk, the WHO has recommended exclusive breastfeeding in COVID-19 suspected/confirmed mother [2]. A strong secretory immunoglobulin A (sIgA) dominant SARSCOV-2 antibody $(\mathrm{Ab})$ response in human breast milk is seen in most COVID-19 mothers [8]. Further studies will determine the protective role of sIgA Ab to SARS-COV-2 in the breast milk of mothers after COVID-19 in protecting their newborn or infant.

A study from Spain on 22 COVID-19 mothers by Pereira et al. found that if adequate hygiene and precautions to prevent respiratory droplet infection are taken by the mother, breastfeeding is safe [9]. Guidelines of Indian Academy of Pediatrics, National Neonatology Forum of India, and the Federation of Obstetric and Gynaecological Societies of India, in cases of mothers, suspected or confirmed COVID-19-positive, recommend rooming-in with mother, and exclusive breastfeeding with precautions such as wearing mask, frequent hand washing, frequent breast washing, routine cleaning, and disinfecting the surfaces. If rooming-in not possible due to sickness in neonate or mother, then expressed breast milk collected hygienically without pasteurization should be given [10].

Breastfeeding Promotion Network of India has advised; in case, the mother is sick and unable to breastfeed directly, she can express her breast milk, which should be given to the infant with 
a clean cup and/or spoon by a healthy caregiver. In a situation when the mother is unable to breastfeed or express breast milk (on ventilator/ICU), as WHO advises, wet nursing, donor human milk, relaxation, or appropriate breast milk substitutes should be used [11].

Distribution of infant formula occurred as part of pandemic relief efforts during India's lockdown. Infant milk substitutes (IMS) were being donated to poor mothers and babies by various Non-Profit Organizations and Government Agencies as a relief measure for the COVID-19 pandemic that the IMS Act of 1992 prohibits the distribution and promotion of IMS for children under the age of 2 years [12].

Breast milk from COVID-19 infected mothers has shown to have sIgA. Breast milk contains several agents which augment the baby's immunity and cognitive development [8]. Anti-SARSCOV-2 IgG and IgA present in COVID-19-positive mothers' milk inactivates the SARS-CoV-2. Hence, breastfeeding is advised in COVID-19-positive mothers [13]. Spatz says in the editorial that health-care providers should use this opportunity of coronavirus pandemic to leverage breastfeeding as a critical intervention to improve health and developmental outcomes and save the lives of children around the world [14].

This was a single service hospital-based study and the small sample size was the study limiting factor. A multicentric study with a larger sample size would enable its wider application.

\section{CONCLUSION}

This study provides a better understanding of prevalent knowledge of healthcare workers regarding the advice to be given about breastfeeding to mothers who are suspected/ confirmed for COVID-19 during confinement or after delivery. This study provides a foundation to focus on the promotion of optimal breastfeeding practices during the COVID-19 pandemic and creating awareness among HCWs about WHO Guidance on Breastfeeding and COVID-19.

\section{REFERENCES}

1. World Health Organization. Breastfeeding and COVID-19: Scientific Brief. Geneva: World Health Organization; 2020. Available from: https://www. who.int/publications/i/item/10665332639. [Last accessed on 2020 Sep 15].

2. World Health Organization. Infant and Young Child Feeding: Fact Sheet. Geneva: World Health Organization; 2020. Available from: https://www. who.int/news-room/fact-sheets/detail/infant-and-young-child-feeding. [Last accessed on 2020 Sep 15].

3. Academy of Breastfeeding Medicine. ABM Statement on Coronavirus 2019 (COVID-19). New York: Academy of Breastfeeding Medicine; 2020. Available from: https://www.bfmed.org/abm-statementcoronavirus. [Last accessed on 2020 Sep 15].

4. Royal College of Obstetricians and Gynaecologists. Coronavirus (COVID19) Infection in Pregnancy. London: Royal College of Obstetricians and Gynaecologists, Royal College of Midwives, Royal College of Paediatrics and Child Health, Public Health England and Health Protection Scotland; 2020. Available from: https://www.rcog.org.uk/globalassets/documents/ guidelines/2020-06-18-coronavirus-covid-19-infection-in-pregnancy.pdf. [Last accessed on 2020 Sep 15].

5. World Health Organization. WHO COVID-19: Case Definitions. Geneva: World Health Organization; 2020. Available from: https://www. who.int/publications/i/item/WHO-2019-nCoV-Surveillance_Case_ Definition-2020.1. [Last accessed on 2020 Sep 15].

6. World Health Organization. Frequently Asked Questions: Breastfeeding and COVID-19: For Health Care Workers. Geneva: World Health Organization; 2020. Available from: https://www.apps.who.int/iris/handle/10665/332719. [Last accessed on 2020 Sep 15].

7. Krejcie RV, Morgan DW. Determining sample size for research activities. Educ Psychol Meas 1970;30:607-10.

8. Fox A, Marino J, Amanat F, Krammer F, Hahn-Holbrook J, Zolla-Pazner S, et al. Evidence of a Significant Secretory-IgA-Dominant SARS-CoV-2 Immune Response in Human Milk Following Recovery from COVID-19. New York: MedRxiv; 2020.

9. Pereira A, Cruz-Melguizo S, Adrien M, Fuentes L, Marin E, Forti A, et al. Breastfeeding mothers with COVID-19 infection: A case series. Int Breastfeed J 2020:15:1-8.

10. Chawla D, Chirla D, Dalwai S, Deorari AK, Ganatra A, Gandhi A, et al. Perinatal-neonatal management of COVID-19 infection-guidelines of the Federation of Obstetric and Gynaecological Societies of India (FOGSI), National Neonatology Forum of India (NNF), and Indian Academy of Pediatrics (IAP). Indian Pediatr 2020;57:536-48.

11. Breastfeeding Promotion Network of India. COVID-19 and Breastfeeding Information Update. Mumbai: Breastfeeding Promotion Network of India; 2020. Available from: https://www.bpni.org/wp-content/uploads/2020/05/ Update-on-COVID-19-and-Breastfeeding.pdf. [Last accessed on 2020 Oct 03].

12. Bhatt N. Breastfeeding in India is disrupted as mothers and babies are separated in the pandemic. BMJ 2020;370:m3316.

13. Pace RM, Williams JE, Järvinen KM, Belfort MB, Pace CD, Lackey KA, et al. COVID-19 and Human Milk: SARS-CoV-2, Antibodies, and Neutralizing Capacity. New York: MedRxiv; 2020.

14. Spatz DL. Using the coronavirus pandemic as an opportunity to address the use of human milk and breastfeeding as lifesaving medical interventions. J Obstet Gynecol Neonatal Nurs 2020;49:225-6.

Funding: None; Conflicts of Interest: None Stated.

How to cite this article: Rajan AR, Vishal AK, Bhave AA. Assessment of knowledge of healthcare workers in a service hospital regarding breastfeeding practices in mothers confirmed or suspected to have COVID-19. Indian J Child Health. 2020; 7(11):437-440. 